Revue des études hébraïques et juives

18 | 2013

Le Proche-Orient ancien à la lumière des sciences sociales

\title{
Carnaval à Soura : Bakhtine et le Talmud de Babylone
}

Carnival at Sura: Bakhtin and the Babylonian Talmud

קרנבל בסורא:בחטין והתלמוד הבבלי

\section{Daniel Boyarin}

Traducteur : Madalina Vârtejanu-Joubert

\section{(2) OpenEdition}

Journals

Édition électronique

URL : https://journals.openedition.org/yod/1787

DOI : $10.4000 /$ yod. 1787

ISSN : 2261-0200

\section{Éditeur}

INALCO

\section{Édition imprimée}

Date de publication : 1 septembre 2013

ISBN : 978-2-85837-207-8

ISSN : 0338-9316

\section{Référence électronique}

Daniel Boyarin, "Carnaval à Soura : Bakhtine et le Talmud de Babylone », Yod [En ligne], 18 | 2013, mis en ligne le 08 juillet 2013, consulté le 08 juillet 2021. URL : http://journals.openedition.org/yod/1787 DOI : https://doi.org/10.4000/yod. 1787

Ce document a été généré automatiquement le 8 juillet 2021.

\section{(c) (†) 8}

Yod est mis à disposition selon les termes de la Licence Creative Commons Attribution - Pas d'Utilisation Commerciale 4.0 International. 


\title{
Carnaval à Soura : Bakhtine et le Talmud de Babylone
}

\author{
Carnival at Sura: Bakhtin and the Babylonian Talmud \\ קרנבל בסורא: בחטין והתלמוד הבבלי
}

\section{Daniel Boyarin}

Traduction : Madalina Vârtejanu-Joubert

\section{NOTE DE L'ÉDITEUR}

Article traduit de l'anglais par Madalina Vârtejanu-Joubert.

Dans sa célèbre et désormais incontournable interprétation de Rabelais, Bakhtine écrivait : «Sa finalité était de défaire la manière ordinaire de percevoir les événements. Il s'est efforcé de les observer d'un œil nouveau, d'interpréter leur contenu tragique ou comique à travers le regard de la foule rieuse des foires. " Fait surprenant, dans le Talmud aussi « le potentiel de sagesse de l'imaginaire populaire » est convoqué pour "défaire le cadre sérieux et étriqué imposé par les classes dominantes »" et ce, justement dans des passages qui visent tous à instaurer un tel cadre sérieux et étriqué, celui du groupe dominant des rabbins. J'entends sans cesse depuis des décennies la phrase suivante : "Si tu n'arrives pas à saisir ce qu'est le Talmud, pense à Rabelais !", prononcée certes avec un brin de provocation. C'est en effet l'œuvre de Rabelais que j'ai toujours tenue pour la plus proche, dans le style et dans l'esprit, du Talmud; la lecture de Bakhtine y est pour beaucoup.

Le Talmud, c'est de la littérature et par conséquent, poser la question de son genre est fondamental dans une démarche littéraire, autrement dit interprétative. Néanmoins, les difficultés surgissent instantanément, car pour définir un genre littéraire il faut pouvoir comparer et opposer, trouver des écrits similaires et des écrits différents ${ }^{2}$.

Une hiérarchie très stricte a été fixée à l'époque post-talmudique entre les débats juridiques - halakha - et les passages narratifs - aggada. Cette hiérarchie a été établie en 
prenant en compte des critères assez proches de la notion de décorum (bienséance), propre à la critique littéraire. Ainsi, seule la halakha serait, pour les rabbins et les sages, digne d'une approche en clé sérieuse. C'est une telle approche qui a dominé les pratiques d'interprétation du Talmud de Babylone depuis ses débuts (à ce propos il faut préciser qu'un écart de plusieurs siècles sépare la date tardive du Talmud de Babylone dans sa forme actuelle et ses premiers commentaires). Depuis le Moyen Âge jusqu'au début de l'époque moderne, on a vu apparaître plusieurs anthologies de aggada talmudique qui extrayaient ces passages de leur contexte halakhique originel. Abstraction faite de cette pratique, il est quand même facile de constater que les commentaires traditionnels négligent totalement les passages aggadiques, et surtout ceux qui portent la marque du grotesque ${ }^{3}$. De grandes autorités ont rédigé des commentaires distincts, l'un sur la partie halakha et l'autre sur la partie aggada. Lorsque dans les éditions imprimées modernes ils ont été rassemblés en un seul texte, le commentaire halakhique apparaissait en gros caractères et le commentaire aggadique en petits. Plus près de nous par contre, et presque dans un élan déconstructiviste, le caractère littéraire de la aggada lui valut fréquemment un statut privilégié, inversant ainsi l'ancienne hiérarchie. La conséquence incontestable de la pratique traditionnelle fut de valoriser la halakha en tant qu'objet sérieux et "sacré » et de délaisser la aggada considérée souvent comme édifiante, mais aussi de temps en temps, comme embarrassante. De fait, comme la distinction entre ces deux « genres » n'est pas du tout indiquée dans le Talmud lui-même, on en a conclu récemment qu'elle sert seulement à opérer ce découpage et qu'elle doit être abandonnée dans la recherche académique 4 . Les vieilles pratiques de lecture talmudique ressemblent à une lecture de Platon à travers le prisme de la philosophie et uniquement de la philosophie. Ce mode de lecture ne peut en aucun cas être ignoré ou délégitimé sans qu'on fasse violence au texte, mais il n'épuise pas le sujet. Encore aujourd'hui la recherche sur le Talmud de Babylone produit principalement des études thématiques ou philologiques. Celles qui se penchent sur les questions de structure et de composition le font surtout dans le cadre de la « haute » critique (des sources) qui cherche à identifier les différentes strates du Talmud de Babylone (par exemple Shamma Friedman ${ }^{5}$ et ses étudiants) et instrumentalise cette approche en vue de la reconstitution historique de ces strates ${ }^{6}$. La composition dans son ensemble est rarement abordée: on pourrait croire que la plupart des chercheurs considèrent, sans le dire explicitement, que le Talmud de Babylone est une composition sui generis. À contre-courant, mon article cherche à mettre en évidence un élément qui apparaît comme un sacrilège : comment dans un seul et unique livre, voire à la même page, une discussion sublime sur la prière côtoie le «naturalisme suburbain » décrit par Bakhtine.

Les lecteurs de Platon comme ceux du Talmud de Babylone sont choqués, si toutefois ils s'autorisent cette expérience, devant toute incongruité qu'ils s'efforcent de censurer ou d'oblitérer. Pourquoi le grand et sacro-saint Socrate se comporte-t-il en bouffon? Pourquoi tel passage sur Dieu et sur la manière de se comporter à la synagogue suit de près quelque chose qui ressemble fortement à une plaisanterie à connotation sexuelle ? La même énigme, ou presque, se cache à l'intérieur de ces deux corpora. D'abord, ce sont deux textes extrêmement sérieux. Comme le disait Whitehead, «la philosophie occidentale n'est qu'une suite de notes de bas de page aux dialogues de Platon ».[ Platon représente pour les penseurs occidentaux la somme exhaustive d'investigation, et même d'instigation, de tous les grands thèmes de la philosophie: l'ontologie, l'épistémologie, l'esthétique et l'éthique. Pour les Juifs traditionnels, le Talmud de 
Babylone constitue la base de la vie religieuse juive, aussi bien en termes de pensée et de spiritualité qu'en termes de pratique. Il est facile, et il l'est depuis des millénaires, de lire les dialogues de Platon comme philosophie et le Talmud de Babylone comme texte légal juif ou comme texte religieux édifiant. La consistance formelle de telles lectures est rassurante et offre à ces communautés de lecteurs le sens de leur intégrité intellectuelle, morale ou religieuse. Mais que fait-on d'un passage où de sublimes assertions spirituelles interfèrent avec le récit des performances sexuelles d'un rabbin (sa capacité, selon un passage, d'avoir des rapports sexuels avec plusieurs vierges sans faire couler de sang)?

Arrivés à ce point, il faut prendre certaines précautions, surtout avec le Talmud de Babylone encore plus éloigné culturellement de nous que Platon. Il est important de ne pas plaquer sur lui des catégories anachroniques ou autrement inappropriées. Ce qui à nos yeux pourrait apparaitre comme un sujet "suburbain " ne semble pas avoir été considéré comme tel par les rabbins babyloniens. Ainsi, on peut même dire qu'une note rabelaisienne se trouve déjà dans la sugya telle quelle et dans les discussions "sérieuses » en matière de halakha. Il ne faut donc pas établir des distinctions trop nettes, selon nos propres catégories culturelles (héritées de la pensée grecque), entre "élévation" et "bassesse ", ni entre la halakha, démarche qui serait régie par les parties supérieures de l'individu, et la aggada, par les parties inférieures. Ceci ne fonctionnerait pas pour de nombreuses raisons que toute personne un tant soit peu familiarisée avec le Talmud peut tout de suite identifier.

Je voudrais néanmoins aller au-delà de cette perspective qui fut aussi la mienne dans de précédentes recherches ${ }^{7}$. Sans méconnaitre cette parenté intellectuelle, mon intérêt se porte à présent sur un autre phénomène, de nature textuelle et littéraire cette fois, et sur sa signification. Ce phénomène, comme je l'ai appelé, se présente sous un double aspect: d'un côté il s'agit tout simplement de la présence de récits qui non seulement célèbrent la partie inférieure du corps humain, mais surtout dépeignent les rabbins les héros du Talmud - sous un jour grotesque, compromettant ou éthiquement douteux. D'un autre côté, et plus important encore, il s'agit du choix littéraire fait dans la Babylonie rabbinique de créer et de se doter d'un seul livre - le Talmud de Babylone - livre dans lequel se trouvent côte à côte des récits mettant en scène les mêmes rabbins de manière contradictoire: comme producteurs du corpus éthique et religieux de la tradition et comme acteurs de récits aggadiques débridés, perturbant l'image du rabbin modèle et de la Torah éternelle et sacrée. Ce phénomène est quasiment unique dans la littérature rabbinique puisque dans les textes palestiniens les genres sont séparés de manière plus accentuée, voire complètement. Le caractère hétérogène du Talmud de Babylone est un fait reconnu: «Beaucoup plus que les compilations rabbiniques palestiniennes, le Talmud de Babylone se montre encyclopédique, car il englobe plus de productions littéraires rabbiniques que n'importe quelle compilation palestinienne datant d'à peu près la même époque. $\aleph^{8}$ Une chose, me semble-t-il, est passée inaperçue jusque-là: le fait que ce caractère multigénérique du Talmud de Babylone le rend très proche des autres corpora littéraires, plus ou moins contemporains et qui thématisent même cette mixité des genres. Quelques commentateurs talmudiques du Moyen Âge ont perçu la nature foncièrement hétérogène du Talmud de Babylone (et c'est même par ce biais qu'ils ont expliqué son nom): «Nous qui étudions le Talmud de Babylone, considérons que celui-ci nous suffit, car il mélange Bible, Michna et Talmud. $»^{9}$ Par cette phrase, le grand Rabbenu Tam, importante autorité talmudique du Nord de la France au XI siècle, 
témoigne de ce sentiment commun que le Talmud de Babylone est un livre tout à fait étrange, un unicum même sur le plan rabbinique, a fortiori sur celui de la littérature universelle, un livre composé de nombreux éléments disparates, tous "mélangés». D'où le jeu de mots balul «mélange » et bavli «babylonien ». En soi, ce jeu de mots tire son origine de la Bible qui interprète la Tour de Babel comme la tour du mélange de langues (la Tour de l'Hétéroglossie) et qu'il faut comprendre sans doute comme la Tour du Babillement. Le Talmud de Babylone est une Tour de Babel hétéroglotte. Cependant, le texte que Rabbenu Tam commente a un sens assez différent : un rabbin d'Erets Israël condamne les Babyloniens (pas le Talmud, mais les Juifs en général) pour leur confusion à propos de la triade Bible, Michna, Talmud. Mais Rabbenu Tam confère à ce passage un sens positif: si le Talmud de Babylone est un mélange, alors le commandement d'étudier ces trois sections de la Torah sera accompli par l'étude d'un seul texte! Cette cacophonie des langues, une Babel aux parlers mélangés, ressemble au grotesque sublime dont l'image emblématique est le hoquet d'Aristophane. La question est ainsi posée : comment allons-nous expliquer ces violations grossières du décorum chez Platon et dans le Talmud de Babylone? On peut aussi deviner la réponse : de par leur forme ces deux corpora s'apparentent au genre explicite du spoudogeloion.

Je vais proposer une formule largement inédite de lecture du Talmud de Babylone, qui ne tranche pas entre le sérieux et le comique, ni ne les annihile selon nos conceptions actuelles. On cherchera au contraire à déceler dans le dialogue que nouent ces éléments à l'intérieur d'un même texte, une manière de production de sens plus complexe à laquelle n'ouvrent pas les deux autres paradigmes de lecture. On peut ainsi constater la simultanéité suivante : la mise en exergue, à travers un protreptique très sérieux, de tout un ensemble de pratiques (intellectuelles, corporelles/politiques) doublée de la contestation de leur validité absolue. Lu ainsi, le Talmud pourvoit des exempla autorisant une interprétation qui va au-delà de la polarité absolutisme-tolérance. Cette nouvelle interprétation est rendue possible par «le surgissement d'un deuxième ton, perçu inéluctablement comme une contradiction flagrante dans la conception de l'auteur $»^{10}$. Bien entendu, dans une œuvre dialogique ou dans toute lecture dialogique d'une œuvre littéraire, c'est justement ce deuxième ton qu'on veut détecter. Bakhtine nous laisse dans une grande ambiguïté quant au statut de cette "contradiction flagrante». Si texte véritablement dialogique il y a, c'est dans la «contradiction flagrante » que se niche le dialogue, dans les différents moments textuels qui rendent possible (ou pas) la contestation interne ou externe d'un système idéologique et qui révèlent la portée d'une telle contestation à l'intérieur d'un ordre culturel donné. Mon analyse regarde le Talmud comme un ouvrage incorporant, quoique de façon non organique, ce type de tons secondaires et de contradictions flagrantes. Pour Bakhtine, le texte qui permet amplement d'y voir une contradiction flagrante dans la pensée de l'auteur est la satire menippéenne.

La satire ménippéenne est connue aussi sous le nom de spoudogeloion, un genre littéraire particulier créé par et pour les intellectuels, dans lequel leurs propres pratiques sont à la fois moquées et mises en exergue. La légende raconte qu'elle a été conçue par un cynique de la première heure, Ménippe de Gadara, au III siècle avant $n$. è. La satire ménippéenne représente une sorte d'usurpation d'identité mettant en scène la façon dont les héros d'une communauté intellectuelle sont dépouillés de leur statut ; pour ce faire, la satire ménippéenne associe genre sérieux et genre comique et donne naissance à un texte qui ne respecte aucun décorum, fait admis déjà depuis l'antiquité. Puisque la force de ce genre est de remettre en question le sérieux et l'autorité des 
pratiques intellectuelles mêmes, il s'agit là, à mes yeux, d'une voie importante qui mène aussi à la compréhension de l'idéologie talmudique. Il ne faut cependant pas perdre de vue que la remise en cause, ou la limitation, de l'efficacité des pratiques intellectuelles ne revient jamais à renier leur autorité.

On considère généralement que le but de la satire est de tourner en dérision une certaine tradition littéraire ou structure socioculturelle, mais ceci est vrai d'un seul type de satire. Il ne faut pas confondre satire ménippéenne et satire en général. La satire ménippéenne est satire dans le sens de satura, un mélange de choses qui ne se ressemblent pas, qui se contredisent les unes les autres - elle n'est aucunement une censure de l'immoralité comme dans la tradition d'Horace ${ }^{11}$.

Dans Meguilla $7 \mathrm{~b}$ on trouve le texte suivant :

Rabbah dit : Le jour de Pourim il faut s'enivrer jusqu'à ne plus savoir [la différence] entre « maudit soit Haman » et « béni soit Mordekhaï ».

Rabbah et Rabbi Zeïra ont fêté Pourim ensemble. [Quand Rabbah fut ivre] il alla et tua Rabbi Zeïra. [Quand il redevint sobre/ le matin] Rabbah pria pour Rabbi Zeïra et le ramena à la vie.

L'année suivante, [Rabbah] dit à [Rabbi Zeïra] : «Que le maître vienne et qu'on fête Pourim [ensemble]. » [Rabbi Zeïra] dit à [Rabbah] : «Les miracles ne se produisent pas tout le temps. $»^{12}$

Bien sûr, ce texte concerne Pourim, le jour par excellence du carnaval. Mais il est aussi emblématique du mélange des genres dans le Talmud. Le discours sérieux sur le devoir, sur la manière d'observer la fête, est combiné au récit comique qui mine l'image des héros de la halakha.

Selon la tradition antique, le sens de satura serait "saucisse", même si de savants chercheurs du siècle dernier - dans leur involontaire moment pantagruélique - se demandaient si c'était bien "saucisse " ou une sorte de pudding. Voici la note de Ullman à ce sujet ${ }^{13}$ :

Ce mot a été compris comme saucisse, par exemple par Friedrich Leo [dans un article de la revue] Hermes. [Zeitschrift für Classische Philologie], xxIv, 70, n., mais un examen attentif des données montre l'absurdité d'une telle définition. Elle est due au fait que les dictionnaires donnent seulement ce sens pour farcimen. Il est évident que celui-ci n'est qu'un sens spécifique parmi d'autres et qu'il devait avoir le sens générique de «farce». Satura ne figure pas sur la liste des saucisses mentionnées par Varron De lingua latina v. 110 f. Curieusement, Fritzsche ([dans son édition de 1875 des] Sermons d'Horace, p. 13) comprend le sens de farcimen chez Varron comme étant celui de lanz. Il s'est de toute évidence fié à sa mémoire en ce qui concerne la recette de Varron. Il n'y a aucune raison de supposer que la recette donnée dans les Questions Plautines ne s'appliquerait pas au genus farciminis, comme le fait Pease dans Harper's Dict. of Class. Lit., à l'entrée « satura ».

Que ce soit une farce, un pudding noir ou une saucisse, le Talmud de Babylone devient beaucoup plus intelligible si on l'appréhende comme une menippea, une tradition légendaire de type carnavalesque mûrie par son terreau culturel ${ }^{14}$. Bakhtine avait déjà énoncé les taxons de ce type d'écrits en se référant, à titre d'exemple, aux récits sur Socrate (à la fois le récit d'Alcibiade et les légendes sur Xantippe) ${ }^{15}$. Il décrit la menippea en ces termes :

En général les légendes de type carnavalesque diffèrent profondément des légendes épiques qui héroïsent - les légendes carnavalesques dévalorisent le héros et le ramènent sur terre, elles le rendent familier, le rapprochent, l'humanisent ; le rire ambivalent de carnaval consume tout ce qui est guindé et rigide, mais en aucun cas ne sape le pivot héroïque, essence de son personnage ${ }^{16}$. 
On aurait pu écrire presque la même chose des légendes biographiques de la littérature rabbinique et encore plus du Talmud de Babylone. Nous y trouvons les héros rabbiniques présentés à la fois dans leur plénitude et leurs embarras corporels, dans leur égoïsme et leur méchanceté et, souvent, dans des situations extrêmes et malencontreuses. Non seulement la aggada mais, assez souvent, la halakha du Talmud de Babylone est elle aussi submergée par le grotesque au sens bakhtinien et rabelaisien du terme. Il y a des débats halakhiques qui abordent par exemple, le sujet d'un homme ayant pénétré son corps de son propre pénis, ou celui de l'objet avec lequel s'essuient les rabbins après défécation, ou enfin, des énoncés d'une grande profondeur tel: « Heureux l'homme dont le cabinet d'aisances est à proximité de sa table. »

L'hybridité, l'incongruité sont le terreau dont s'est nourrie la littérature hellénistique postclassique. Ce qui définit les écrits de l'hellénisme tardif ( $\mathrm{II}^{\mathrm{e}}-\mathrm{VI} \mathrm{e}^{\mathrm{e}}$ siècles) par rapport aux précédents genres et registres linguistiques ${ }^{17}$, est l'absence de décorum. Comme l'a bien montré Bakhtine, cette période connait un renouveau littéraire et culturel qui s'exprime à travers des formes textuelles comme la satire ménippéenne, les dialogues parodiques de Lucien et le roman antique. Les Anciens ne reconnaissaient le spoudogeloion que dans l'œuvre de Ménippe (cf. Strabon 16.2.29), par conséquent l'emploi par Bakthine des deux termes, spoudogeloion et satire ménippéenne, est virtuellement synonymique. Il faut admettre cependant que l'existence d'un "genre » distinct appelé satire ménippéenne n'est pas formellement attestée, du moins pas avant la Renaissance. Il se peut en effet que la satire ménippéenne n'ait pas tant été un genre formel qu'une disposition littéraire et le reflet de certaines habitudes de pensée. Genre distinct ou pas, la manière dont Bakhtine l'a abordée ouvre des possibilités heuristiques extrêmement incitantes et permet une approche renouvelée du Talmud. Avec l'Icaromenippus de Lucien, la satire ménippéenne semble s'affirmer comme "l'enveloppe formelle la plus à même de figurer les querelles et les débats des savants ${ }^{18}$. Il n'est donc pas surprenant de trouver des affinités entre le Talmud et ce que Bakhtine appelle menippea.

La culture dans laquelle s'origine le Talmud se caractérise, grosso modo et inter alia, par le fait de glorifier sa dépendance envers l'investigation rationnelle comme voie de vérité. Par conséquent, la «dialogisation" d'une telle culture viendra logiquement d'une forme antithétique qui ne représente plus ses héros comme des êtres entièrement rationnels. Un des moyens pour y parvenir est de mettre à profit la forme sério-comique pour créer un corpus littéraire où la démarche la plus sérieuse et la plus raisonnable côtoie les aspects les plus « bas » et les plus absurdes des personnages et de leurs vies privées. Pour résumer en mes propres termes la position bakhtinienne, cela revient à dire qu'un genre folklorique oral (terme lui aussi problématique en soi) a été formalisé et couché par écrit. Bakhtine a avancé l'idée que Platon s'est approprié une forme polyphonique orale, le dialogue, en la convertissant en une forme écrite de monologue, la dialectique. En même temps, toujours selon Bakhtine, on voit comment la structure du récit comique des dialogues incorpore dialogiquement le dialogisme de type plutôt carnavalesque de la forme antérieure. La forme, et Bakhtine ne le dit pas assez clairement, constitue une dialogisation du propre monologue de Platon (Bon gré mal gré ? Je ne le crois pas, Platon était trop rusé pour cela) ${ }^{19}$.

Platon se trouve donc, selon cette hypothèse, aux fondements (mais pas forcément à l'origine) d'un mode de production textuelle présent dans le Talmud de Babylone de manière beaucoup plus explicite et prolixe. Selon ce mode, les moments essentiels et 
très sérieux où s'exerce le raisonnement dialectique, sous forme de dialogue (ou plutôt de pseudo-dialogue), sont intégrés dans une structure comique qui questionne, du moins en apparence, le sérieux du contenu de la dialectique elle-même, mais ne donne pas la réponse à la question posée. Autrement dit, il interroge, mais ne rejette d'aucune manière la «sériosité » du « sérieux ». Cette façon de lire le Talmud de Babylone en tant que récit comique contenant du hautement sérieux, pourrait paraitre une exagération injustifiée. J'admets ce point, mais je tiens néanmoins à suggérer que si nous lisons le Talmud de Babylone de cette manière, c'est pour découvrir des choses qui ne se révèlent pas à travers un autre type de lecture. Je pourrais comparer la méthode de lecture que j'adopte ici à la manière dont on perçoit l'image du canard-lapin : on peut passer volontairement d'une Gestalt (forme) à l'autre, mais on ne peut jamais voir les deux en même temps. C'est aussi le cas dans le cas du Talmud de Babylone, notre perception alterne - on voit une fois la Gestalt sério-comique talmudique, mais on retourne aussi en arrière pour voir son immense sérieux. Sans ce double mouvement on perd beaucoup.

Le dialogue reproduit chez Platon comme dans le Talmud est lui-même dialogique, incorporant toutes les voix en une seule conscience, celle de l'« auteur ». Cet imbroglio est généré par l'apparent (plus qu'ostensible, mais certainement pas absolu) engagement de l'« auteur ", Platon ou le stama (auteur(s)/rédacteur(s) anonyme(s) du Talmud), envers le sérieux de cette dialectique même. C'est là l'objectif de ces textes : convaincre le lecteur de la justesse de leur très peu dialogique vision du monde. Pourquoi alors ces " auteurs " (une fonction textuelle dans les deux cas) placeraient-ils si ostensiblement leurs propres pratiques textuelles dans un contexte aussi irrévérencieux? Je ne peux évidemment pas décrire les «intentions » derrière ces artifices textuels, mais je voudrais suggérer que les effets de ces artifices peuvent être compris comme une réflexion de second plan sur les fondements épistémologiques de ces très sérieuses pratiques. C'est comme si le militant, philosophe ou docteur de la loi, se disait un matin (voire tout le temps) : «Et si je me trompais?» Cette perpétuelle voix autocritique, une voix du dialogue, dispute la voix de l'engagement militant qui est le ton dominant du texte, permettant ainsi de contempler l'abysse en même temps que l'énonciation appuyée des pratiques permettant d'éviter d'y tomber (la philosophie, la Torah).

\section{NOTES}

1. Mikhail Bakhtin, Rabelais and His World, trad. Hélène Iswolsky, Bloomington, Indiana UP, 1984, p. 439. Édition française: L'œuvre de François Rabelais et la culture populaire au Moyen Âge et sous la Renaissance, Paris, Gallimard, 1970 (1 ${ }^{\text {re }}$ éd. russe 1965).

2. David Kraemer, Reading the Rabbis: The Talmud as Literature, New York, Oxford UP, 1996, p. 7.

3. Au sujet de telles stratégies dans l'œuvre de Rabelais, voir M. Bakhtin, Rabelais, p. 134-136.

4. Barry Wimpfheimer, Narrating the Law: A Poetics of Talmudic Legal Stories, Philadelphie, U. of Pennsylvania P., 2011. Cela ne signifie certainement pas que cette distinction soit dépourvue de tout fondement. Dans Baba Qamma 60b le Talmud raconte une charmante histoire au sujet d'un 
maître ayant deux disciples. L'un voulait apprendre des traditions halakhiques [shema'tata] et l'autre de la aggada. Aucun ne laissait le maître enseigner l'autre genre. Le maître leur raconta l'histoire d'un homme qui avait deux femmes, une jeune et une vieille. La jeune lui arrachait tous les cheveux blancs, l'autre tous les cheveux noirs jusqu'à ce qu'il soit devenu « chauve de tous les côtés ». De la même manière, ces disciples seraient ainsi privés du savoir de la Torah. Le maître a intelligemment trouvé une manière de leur enseigner à la fois la halakha et la aggada. Une certaine distinction était donc admise. Néanmoins, Wimpfheimer a vu juste quand il affirme que la distinction entre ce que nous appelons aujourd'hui halakha et aggada ne correspond pas aux catégories du texte talmudique. Nous avons toutes les raisons de croire que ce que nous appelons aggada signifie en fait dans le Talmud, comme ici dans Baba Qamma, interprétations homilétiques de la Torah et non pas récits biographiques ; ceux-ci, autant que je sache, ne sont jamais désignés en tant que aggada dans la littérature rabbinique classique.

5. Shamma Friedman, "A Critical Study of Yevamot X with a Methodological Introduction" in H. Z. Dimitrovsky (éd.), Texts and Studies, Analecta Judaica I, New York, The Jewish Theological Seminary of America, 1977, p. 227-441; Talmud 'arukh pereq ha-sokher et ha-ummanin: Bavli Bava Metsi'a pereq shishi: Mahadurah 'al derekh ha-mehqar 'im perush ha-sugyot, Jérusalem, The Jewish Theological Seminary of America, 1990.

6. Par exemple Jeffrey L. Rubenstein, The Culture of the Babylonian Talmud, Baltimore, Johns Hopkins University, 2003 ; Richard Kalmin, Jewish Babylonia Between Persia and Roman Palestine, New York, Oxford UP, 2006.

7. Daniel Boyarin, "The Great Fat Massacre: Sex, Death and the Grotesque Body in the Talmud" in H. Eilberg-Schwartz (éd.), People of the Body: Jews and Judaism from an Embodied Perspective, Albany NY, SUNY Press, 1992, pp. 69-102.

8. Richard Kalmin, "The Formation and Character of the Babylonian Talmud" in S. T. Katz (éd.), The Cambridge History of Judaism: Vol. IV The Late Roman-Rabbinic Period, Cambridge, Cambridge UP, 2006, p. 841.

9. Tosafot sur Avoda Zara 19b.

10. Mikhail Bakhtin, Problems of Dostoevsky's Poetics, éd. et trad. Caryl Emerson, Minneapolis, U of Minnesota P, 1984, p. 82.

11. Joel C. Relihan, Ancient Menippean Satire, Baltimore, Johns Hopkins UP, 1993, p. 20. Voir aussi M. Bakhtin, Rabelais, p. 84 : « La parodie médiévale, surtout avant le $\mathrm{xII}^{\mathrm{e}}$ siècle, ne s'intéressait pas aux aspects négatifs, ni aux imperfections de certains cultes, ni à l'ordre ecclésiastique, ni aux lettrés qui auraient pu être objet de dérision et de destruction. Pour les parodistes médiévaux tout, sans exception, était comique. Le rire était tout aussi universel que le sérieux. »

12. Traduction modifiée à partir de Wimpfheimer.

13. B. L. Ullman, "Satura and Satire ", Classical Philology 8, n 2, 1913, p. 172-194 : "This has been described as a sausage, e.g., Leo, Hermes, xxIv, 70, n., but a consideration of the materials used shows the absurdity of such a definition. It is due to the fact that the dictionaries give this meaning alone for farcimen. It is evident that this is but one of the special meanings of the word, and that it must have had a general meaning stuffing. Satura is not in the list of sausages mentioned by Varro L.L. v. 110 f. Fritzsche (Horace Serm., p. 13) curiously takes Varro's farcimen to be the same as the lanz. He was evidently trusting his memory for Varro's recipe. There is no reason for assuming that the recipe given in the Plautine Questions does not apply to the genus farciminis, as Pease assumes in Harper's Dict. of Class. Lit., S.U. 'satura'." (p. 176, n. 2)

14. Le terme menippea, différent de la « satire ménippéenne ", a été, je crois, inventé par Bakhtin, en faisant référence à une notion qui me semble très importante, celle d'un ensemble d'éléments ménippéens puisés dans différents genres et sources et évoluant ensuite en fonction du temps et de l'espace.

15. M. Bakhtin, Problems of Dostoevsky's Poetics, p. 132.

16. Ibid., p. 132-133. 
17. Pour le concept générique d'hellénisme tardif, voir G. W. Bowersock, Hellenism in Late Antiquity, Jerome Lectures, Eighteen, Ann Arbor, The U of Michigan P, 1990, même si cet ouvrage ne traite pas particulièrement des caractéristiques que j'aborde.

18. Joel C. Relihan, "Menippus in Antiquity and the Renaissance" in B. R. Branham, M.-O. Goulet-Cazé (éds),_The Cynics: The Cynic Movement in Antiquity and Its Legacy, Berkeley \& Los Angeles, U of California P, 1997, p. 282.

19. Je suis parvenu à cette conclusion indépendamment et par d'autres voies que celles suivies par ma collègue Leslie Kurke qui, dans un article remarquable, "Plato, Aesop, and the Beginnings of Mimetic Prose", Representations 94, 2006, pp. 6-52, étayait la thèse du développement de la prose mimétique platonicienne à partir d'une tradition ésopique (reconstituée à partir de sources postérieures), Ésope étant la figure emblématique de l'auteur « mineur ». Le livre de Kurke, sous presse à l'heure où j'ai achevé cet article, va, je présume, considérablement améliorer mes suggestions actuelles.

\section{RÉSUMÉS}

En faisant appel à la théorie dialogique de Mikhail Bakhtine, je me propose d'analyser ici un phénomène à double face : d'un côté il s'agit tout simplement de la présence de récits qui non seulement célèbrent la partie inférieure du corps humain, mais surtout dépeignent les rabbins les héros du Talmud - sous un jour grotesque, compromettant ou éthiquement douteux. D'un autre côté, il s'agit du choix littéraire fait dans la Babylonie rabbinique de créer et de se doter d'un seul livre - le Talmud de Babylone - livre dans lequel se trouvent côte à côte des récits mettant en scène les mêmes rabbins de manière contradictoire : comme producteurs du corpus éthique et religieux de la tradition et comme acteurs de récits aggadiques débridés, perturbant l'image du rabbin modèle et de la Torah éternelle et sacrée.

Using the Mikhail Bakhtin dialogical theory, my concern here is with a double-faced phenomenon: one face is simply the presence of narratives that celebrate the lower body and actively portray the Rabbis in grotesque, compromising, or ethically problematic light. The second face is the literary choice to produce and have only one book in rabbinic Babylonia, the Babylonian Talmud, in which precisely cheek by jowl we find the same Rabbis as the producers of all that is ethical, religious, and fine in the tradition. 
INDEX

Mots-clés : imagination dialogique, spoudogeloion, Talmud de Babylone, Bakhtine Mikhaïl (1895-1975)

Index géographique : Méditerranée, Proche-Orient

\section{מילות מית}

המזרח התיכון: - n - n - n

Keywords : dialogic imagination, spoudogeloion, Babylonian Talmud, Bakhtine Mikhail (1895-1975), Mediterranean, Middle East 